\title{
PTCH1 Gene Deletion
}

National Cancer Institute

\section{Source}

National Cancer Institute. PTCH1 Gene Deletion. NCI Thesaurus. Code C133679.

A molecular abnormality referring to the loss of at least one copy of the PTCH1 gene. 